\title{
Design and Analysis of Two Types of Variable Stiffness Actuator Based on Adjustable Moment Arm Mechanism
}

\author{
Jishu Guo and Guohui Tian \\ School of Control Science and Engineering, Shandong University, 73 Jingshi \\ Road Jinan, Shandong Province, 250061, P. R. China \\ guojishu_2005@163.com,g.h.tian@sdu.edu.cn
}

\begin{abstract}
This paper introduces the design and analysis of two types of novel inherently compliant actuators. The two actuation systems are characterized by the property that the output apparent stiffness can be varied independently from the position. The two proposed actuators can regulate the stiffness through the adjustable moment arm mechanism with minimum energy consumption. In the first VSA, this behavior is realized by implementing a lever arm mechanism with variable pivot point position. Compared with the existing VSA that based on the lever arm principle, the way of the spring compressed is more convenient to be implemented by using the tendon and pulley mechanism. Furthermore, the kinematic structure for spring compression is more beneficial for dealing with the unexpected dynamic collision situation. The internal elastic elements of the VSA use the fitted quadratic spring, which improve the characteristics of actuating torque and output stiffness. The stiffness regulation of the second VSA is realized by implementing the connecting rod and slider mechanism. This is a equivalent model of the lever arm mechanism with a variable length moment arm. The stiffness of the VSA is only related to the effective working radius for linear spring configuration, but not to the applied external torques or the deflection angle. This feature makes the stiffness control of the VSA more convenient. The working principles of the two actuators are elaborated and the characteristics of torque and stiffness of the VSA are presented. The mechanical solutions of the two actuators are described.
\end{abstract}

Keywords: Variable Stiffness Actuators, Adjustable Moment Arm Mechanism, Stiffness And Torque, Mechanical Structure Solutions

\section{Introduction}

In recent years, the interest in variable stiffness actuation system is growing due to the change of trends in robotic applications. Compared with the stiff actuators, the compliant actuators are strongly recommended when robots coexist with human beings or physically interact with them. The compliant actuators can be classified into two main categories: active and passive. Generally, the active compliant actuators are relying on the proper sensors. These sensors increase the cost, and the existence of a response delay is not suitable for dealing with highly dynamic situations in the environment. In the passive compliant actuators, the internal mechanical elastic elements not only ensure the safety of human-robot interaction, but can also be used to store and release the kinetic energy coming from the link's inertia [1].

Among the compliant actuators, many variable stiffness actuators have been recently developed by the robotics community. The variable stiffness actuators are characterized by the ability that the apparent stiffness at the actuated load can be varied independently from its position. The optimal criteria of the variable stiffness actuators are as follows: (i) Stiffness should be changed without energy consumption. (ii) Energy 
efficiency of stiffness adjustment should be $100 \%$. (iii) No energy should be required to maintain the stiffness. (iv) Stiffness should be independent from the external load. (v) Range of stiffness should be unlimited. (vi) No inertia should be added to the output link due to the stiffness adjustment mechanism. (vii) Maximum capacity of the energy storage should be accessible [2].

In order to achieve the features described above, many research efforts have been put into the development of the VSA. Some designs rely on changing the pretension of the internal elastic elements, e.g., the VSA-II [3] and MACCEPA 2.0 [4]. In other designs, which are realized by a variable transmission ratio between the output and the internal springs, have the advantage in reducing the energy/power required to regulate the stiffness. The HDAU [5], AwAS [6], AwAS-II [7], CompAct-VSA [8], CompActARS [9], the vsaUT [10], the vsaUT-II [11], and the mVSA-UT [12] use a lever arm of variable effective length to realize the variable transmission ratio and regulate the apparent output stiffness. As elaborated in [13] and [14] by means of a port-based approach, these actuators have the property that the stiffness can be changed without any energy injection into or extraction from the internal springs. This is an energy efficiency way to regulate the stiffness.

This paper presents two types of variable stiffness actuators based on adjustable moment arm mechanism. The apparent output stiffness can be varied by changing the transmission ratio between the internal elastic elements and the actuator output. The main novelty of the first VSA is the kinematic structure to compress the spring and the using of the fitted quadratic spring group. The novelty of the second VSA is the application of the connecting rod and slider mechanism for changing the effective working radius. This paper is organized as follows. Section 2 describes the working principle of the first VSA. Section 3 presents the design of the fitted quadratic spring and illustrates the characteristics of torque and stiffness of the first VSA. Section 4 describes the mechanical structure solution of the first VSA. Section 5 demonstrates the working principle of the second VSA. The characteristics of torque and stiffness of the second VSA are illustrated in Section 6 while the mechanical structure solution is described in Section 7. Finally, conclusions are drawn in Section 8.

\section{Working Principle of the First VSA}

\subsection{Conceptual Layout}

The actuator discussed in this section is based on the use of a lever arm mechanism with a variable pivot point position. A simple schematic is shown in Figure 1. In this figure, the output disk (1) and the driving disc (3) is coaxially connected by the 4-point contact bearing (2) .The stiffness motor mounted on the driving disc (3) drives the pivot (7) by the bevel gear and threaded shaft mechanism. The (7) can be moved along the radial direction of the (3), thereby changing the moment arm of the lever. The rotatable connection point (4) of the lever (8) is connected with the output disc (1), and a roller (9) is installed on the other end of the lever (8) (whose back has a moving guide groove for the pivot).

Figure 1(a) demonstrates the initial state of the VSA. As is shown in Figure 1(b), when the external torque is applied to the (1), the (8) will be rotated around the (7). Meanwhile, the roller contacts and stretches the tendon. The two ends of tendon (5) or (6) are respectively connected to the spring group pedestal (12). The (12) will be moved along the spring group pedestal guide groove (10), and the spring will be compressed. By calculating the vertical force applied by the tendon (5) or (6) on the (9), we can calculate the torque and stiffness of the actuator. As is shown in Figure 1(b), Figure 1(c), and Figure 1(d), when the pivot point is moved from $\mathrm{H}$ to $\mathrm{H}^{\prime}$ along the radial direction of the (3), in the same deflection angle $\beta$ of the VSA, the amount of deformation of the spring is changed from $\Delta \mathrm{L}$ to $\Delta \mathrm{L}^{\prime}$, i.e., $\mathrm{H}<\mathrm{H}^{\prime}$ and $\Delta \mathrm{L}<\Delta \mathrm{L}^{\prime}$. The 
amount of deformation of the spring, the vertical force on the end point of the lever, and the proportion of the two moment arms are changed. The output stiffness can be regulated by moving the (7) and do not need to apply preload force on the spring.
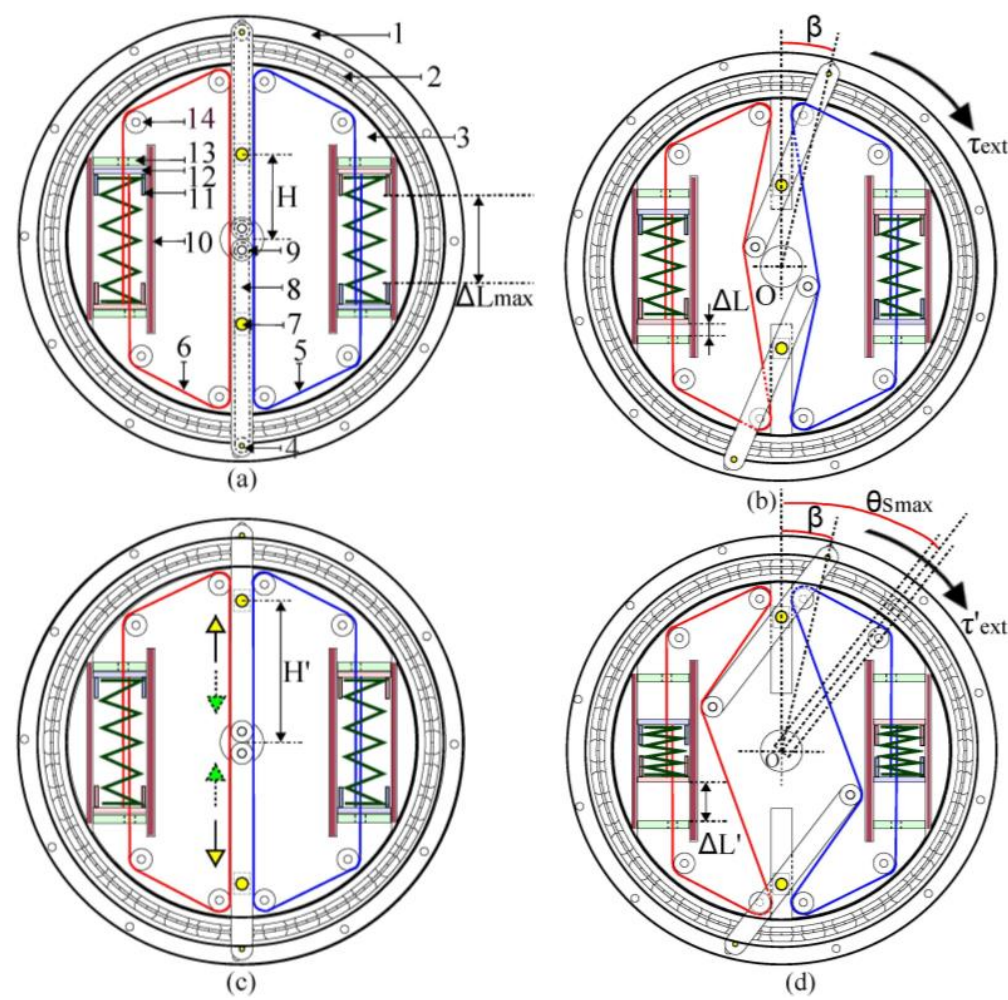

Figure 1. Conceptual Diagram Of The First VSA: (A) Initial State. (B) Passively Rotated By The Exerted Torque. (C) Stiffness Control. (D) Passively Rotated After The Stiffness Adjustment.

The (11) is used to ensure the stability of the spring during working. The (13) represents the fixed baffle for the spring group pedestal and (14) is the diverting pulley for the tendons. The maximum distance between the two spring group guide sleeves is $\Delta \mathrm{L}_{\max }$, and the maximum deflection angle is $\theta_{\text {smax }}$ (mechanical stops by the spring group guide groove mounted on the driving disc (3)). When the two spring group guide sleeves (11) contact with each other, or the maximum deflection angle $\theta_{\text {smax }}$ occurs, or the pivot (7) is moved to the (4) of the lever (8), the actuator will into the stiff operation mode from the compliance mode.

\subsection{Mathematical Modeling}

The working principle of the first VSA is demonstrated with more details in Figure 2. Suppose that the lever arm is at its equilibrium position and the applied external torque $\tau_{\text {ext }}$ rotates the lever arm with an angle $\theta$ with respect to the pivot (D). As a result, the end point $\mathrm{O}^{\prime}$ of the lever $\left(\mathrm{BO}^{\prime}\right)$ has an angle $\theta_{\mathrm{S}}$ with respect to the center of rotation of the actuator. Another end point (B) of the lever contacts with the tendon and compresses the spring. Point $A$ and point $C$ represents the fixed diverting pulley for the tendon. $\mathrm{H}$ is the distance from the pivot (D) to the center of rotation of the actuator $(\mathrm{O})$. The deflection angle $\theta_{\mathrm{S}}$ of the elastic transmission is defined as the difference between the generalized coordinates of the output link and the position motor. 


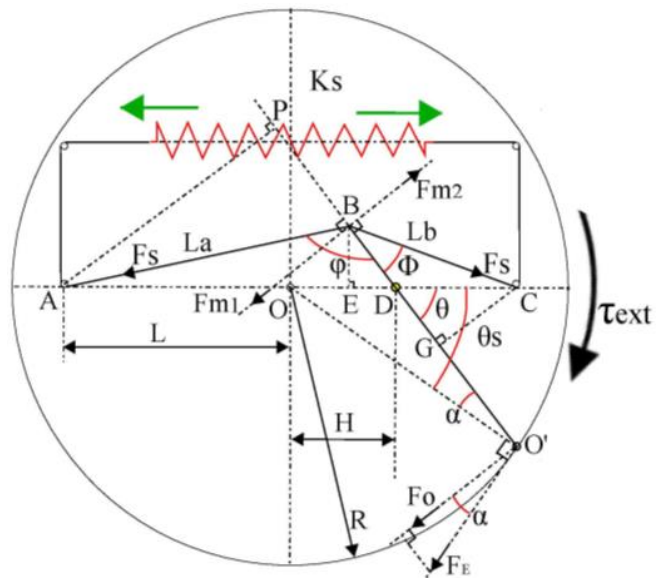

Figure 2. Schematic Description Of The First VSA

Suppose that the elastic elongation of the tendon is neglected, and the spring stiffness is $\mathrm{K}_{\mathrm{s}}$. The parameters definition and calculation formulas are as follows.

$$
\begin{aligned}
& \overline{O O^{\prime}}=\overline{B O^{\prime}}=H_{0}=R>L=\overline{A O}=\overline{O C} \\
& \overline{O D}=H \\
& H^{2}+R^{2}-2 H R \cos \theta_{S}={\overline{D O^{\prime}}}^{2} \\
& \overline{D O^{\prime}}=\sqrt{H^{2}+R^{2}-2 H R \cos \theta_{S}} \\
& \overline{B D}=H_{0}-\overline{D O^{\prime}}=H_{0}-\sqrt{H^{2}+R^{2}-2 H R \cos \theta_{S}} \\
& \angle O O^{\prime} D=\alpha=\arccos \frac{R-h \cos \theta_{S}}{\sqrt{H^{2}+R^{2}-2 H R \cos \theta_{S}}} \\
& \theta=\theta_{S}+\alpha \\
& \overline{B E}=\left(H_{0}-\sqrt{H^{2}+R^{2}-2 H R \cos \theta_{S}}\right) \sin \theta \\
& \overline{A E}=L+H-\overline{B D} \cos \theta \\
& \overline{E C}=L-H+\overline{B D} \cos \theta \\
& L_{a}=\sqrt{\overline{A E}^{2}+\overline{B E}^{2}} \\
& L_{b}=\sqrt{\overline{B E}^{2}+\overline{E C}^{2}}
\end{aligned}
$$

The spring is displaced by $\Delta \mathrm{L}=\mathrm{L}_{\mathrm{a}}+\mathrm{L}_{\mathrm{b}}-2 \mathrm{~L}$ and consequently generates a force $\mathrm{F}_{\mathrm{S}}$ applied in point (B) given by $\mathrm{F}_{\mathrm{S}}=\mathrm{K}_{\mathrm{S}} \Delta \mathrm{L}$. The resultant force $\mathrm{F}_{\mathrm{m}}$ along the perpendicular to $\left|\mathrm{BO}^{\prime}\right|$ applied in point $(\mathrm{B})$ is calculated as follows.

$$
F_{m}=F_{m 1}-F_{m 2}=F_{S} \sin (\pi-\varphi)-F_{S} \sin \phi=F_{S} \sin \varphi-F_{S} \sin \phi=F_{S} \frac{(L+H) \sin \theta}{L_{a}}-F_{S} \frac{(L-H) \sin \theta}{L_{b}}
$$

As a consequence, the elastic torque $\tau_{E}=2 F_{E} R$ which counterbalances the exerted torque is

$$
\tau_{E}=2 \frac{F_{O}}{\cos \alpha} R=2 \frac{F_{m} \overline{B D}}{\overline{D O^{\prime}} \cos \alpha} R .
$$

Finally, the output stiffness of the first VSA is formulated as follows. 


$$
K=\frac{\partial \tau_{E}}{\partial \theta_{S}}
$$

\section{The Characteristics of Torque and Stiffness Of The First VSA}

To regulate the stiffness, the pivot should be moved along the guide groove of the lever. When the pivot is located relatively close to the center of rotation of the actuator, the torque will first increase and then decrease with the increase of the deflection angle. Furthermore, stiffness of the VSA abruptly drops if the external torque exceeds a certain threshold value. This feature has demonstrated in this section by calculation. Therefore, unexpected collision safety can be achieved when the VSA works in human environments, but not beneficial to withstand more external torque load. In order to improve the operating characteristics of the VSA, the fitted quadratic spring was used to replace the general linear spring. Simulation analysis in this section has demonstrated that the fitted quadratic spring can improve the torque load characteristics of the actuator.

\subsection{Spring Design Criteria}

Generally, the commercial availability of nonlinear springs with quadratic force-length relationships is extremely limited. The reliably manufacture of the purely quadratic springs is relatively difficult. The nonlinear spring constructed by a special profile frame and standard linear springs often has a relatively large size, or the special profile frame may require to be designed and manufactured [15]. The compression springs with different height can be combined into a spring group in the form of parallel-type. Each spring has a different deformation amount when the axial load is applied to the spring group. This combination spring group has a nonlinear relationship between the force and displacement. Therefore, the springs with different height and stiffness can be used to constitute a spring group with an arbitrary stiffness.

In this section, the general cylindrical helical compression springs with different parameters are used to constitute an approximate quadratic spring. The end portion of the spring is flat and be tighten. The number of the supporting coil is one. The range of the helix angle $\alpha$ of the spring is recommended from $5^{\circ}$ to $9^{\circ}$. The workspace of the linear spring is recommended from $20 \%$ to $80 \%$ of the allowable maximum deformation. In order to ensure the stability during operation, the spring guide sleeve is adopted. The spring material is piano wire, and $\mathrm{D}$ grade. The main design criteria for the linear compression spring can be seen in [16].

\subsection{Piecewise Linear Spring Group}

By doing the trade-off between mechanical dimensions and performance, the suitable parameters for the springs have been identified and presented in Table 1 . The number of the springs is 16 . We set a different initial height for each spring inside the spring group pedestal. The working distance between the two adjacent springs is $1 \mathrm{~mm}$. The maximum deformation of the spring group is $16 \mathrm{~mm}$. When the both sides of the spring group guide sleeves contact with each other, the springs could not be continued compressed.

In order to reduce the friction between the spring group pedestal and the guide groove, and to ensure the spring group pedestal can withstand a relatively uniform pressure, the arrangement of the springs in the spring group pedestal is presented in Table 2. 
Table 1. Specifications of the Compression Springs Group

\begin{tabular}{|c|c|c|c|c|c|c|c|}
\hline$D^{I}$ & $\begin{array}{l}\mathrm{d}(m \\
m)\end{array}$ & $\begin{array}{l}\mathrm{D}(m \\
m)\end{array}$ & $\mathrm{n}$ & $\mathrm{k}(\mathrm{N} / \mathrm{mm})$ & $\begin{array}{l}\mathrm{t}(m \\
m)\end{array}$ & $\bar{\alpha}$ & $\begin{array}{l}\begin{array}{l}\mathrm{H}_{0}(m \\
m)\end{array}\end{array}$ \\
\hline 1 & 0.5 & 3.5 & $0^{3}$ & 0.4798 & 1.71 & $8.8^{\circ}$ & 52 \\
\hline 2 & 0.6 & 3.5 & $0^{3}$ & 0.995 & 1.64 & $8.5^{\circ}$ & 50 \\
\hline 3 & 0.6 & 3.5 & $0^{3}$ & 0.995 & 1.57 & $8.1^{\circ}$ & 48 \\
\hline 4 & 0.6 & 3.5 & $0^{3}$ & 0.995 & 1.47 & $7.6^{\circ}$ & 45 \\
\hline 5 & 0.6 & 3.5 & $0^{3}$ & 0.995 & 1.37 & $7.1^{\circ}$ & 42 \\
\hline 6 & 0.6 & 3.5 & $0^{3}$ & 0.995 & 1.3 & $6.8^{\circ}$ & 40 \\
\hline 7 & 0.6 & 3.5 & $0^{3}$ & 0.995 & 1.24 & $6.4^{\circ}$ & 38 \\
\hline 8 & 0.6 & 3.5 & $0^{3}$ & 0.995 & 1.14 & $5.9^{\circ}$ & 35 \\
\hline 9 & 0.6 & 3.5 & $0^{3}$ & 0.995 & 1.04 & $5.4^{\circ}$ & 32 \\
\hline $0^{1}$ & 0.6 & 4 & $0^{2}$ & 0.9998 & 1.46 & $6.6^{\circ}$ & 30 \\
\hline $1^{1}$ & 0.6 & 4 & $0^{2}$ & 0.9998 & 1.36 & $6.2^{\circ}$ & 28 \\
\hline $2^{1}$ & 0.6 & 4 & $0^{2}$ & 0.9998 & 1.26 & $5.7^{\circ}$ & 26 \\
\hline $3^{1}$ & 0.6 & 5 & $.5^{1}$ & 0.9751 & 2.2 & $8^{\circ}$ & 24 \\
\hline $4^{1}$ & 0.6 & 5 & $.5^{1}$ & 0.9751 & 2.1 & $7.3^{\circ}$ & 22 \\
\hline $5^{1}$ & 0.6 & 5 & $.5^{1}$ & 0.9751 & 1.82 & $6.6^{\circ}$ & 20 \\
\hline $6^{1}$ & 0.6 & 5 & $.5^{1}$ & 0.9751 & 1.72 & $6.3^{\circ}$ & 19 \\
\hline
\end{tabular}

Table 2. Arrangement of the Compression Springs Group

\begin{tabular}{cccc}
\hline \hline Column & Column & Column & Column \\
1 & 2 & 3 & 4 \\
\hline 7 & 13 & 16 & 6 \\
9 & 1 & 4 & 12 \\
11 & 3 & 2 & 10 \\
5 & 15 & 14 & 8
\end{tabular}

The relationship between force $\mathrm{F}(x)(N)$ and displacement $x(\mathrm{~m} m)$ of the spring group can be expressed as follows.

$$
F(x)= \begin{cases}k_{1} x & 0<x \leq 1 \\ k_{1} x+k_{2}(x-1) & 1<x \leq 2 \\ k_{1} x+k_{2}(x-1)+k_{3}(x-2) & 2<x \leq 3 \\ \cdots \cdots & \cdots \cdots \\ k_{1} x+k_{2}(x-1)+\cdots+k_{16}(x-15) & 15<x \leq 16\end{cases}
$$

By using the least squares method, the best-fit equation for the fitted quadratic spring is obtained such as 


$$
F(x)=0.4965 x^{2}-0.003889 x-0.03237 .
$$

Equation (7) can be simplified as $F(x)=0.4965 x^{2}$. Figure 3 shows that the force difference between the fitted quadratic spring and the actual piecewise linear spring group is very small, i.e., the maximum difference of the two output force is $\Delta \mathrm{F}_{\max }=0.1398 N$. Compared with the piecewise linear spring group, the difference of output torque caused by the fitted spring is also very small. When the position of the pivot is set to be $35 \mathrm{~mm}, 40 \mathrm{~mm}, 45 \mathrm{~mm}$, and $50 \mathrm{~mm}$, respectively, the maximum torque difference caused by the piecewise linear spring group and the fitted quadratic spring is $0.0303 \mathrm{Nm}$. Therefore, the characteristics of actuating torque and output stiffness of the first VSA can be calculated by using the fitted quadratic spring.
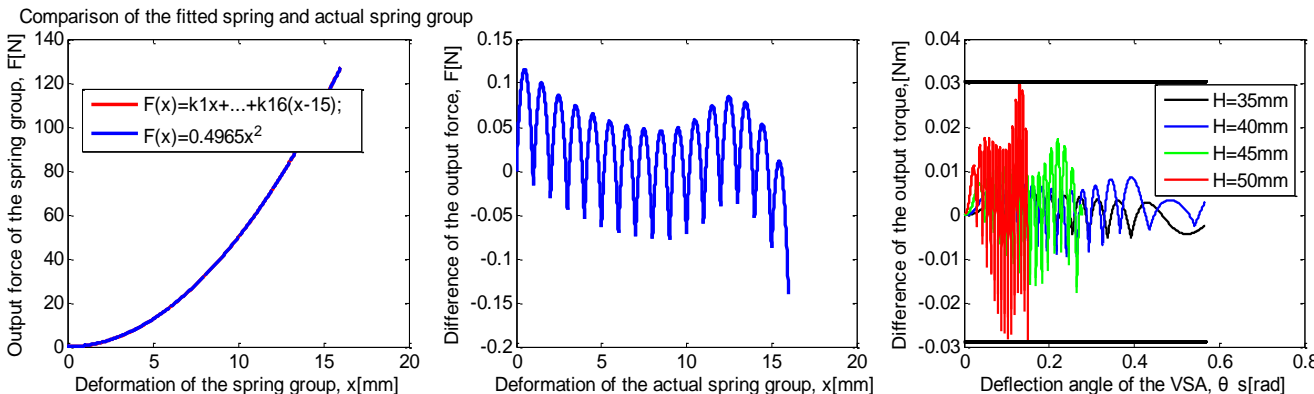

Figure 3. Working Characteristics of the Piecewise Linear Spring Group

\subsection{Characteristics of Torque and Stiffness of the First VSA}

The maximum deflection angle achievable by the elastic transmission is constrained to be $\pm 0.5674 \mathrm{rad}$. In this section, the value of $\mathrm{L}$ and $\mathrm{R}$ are defined as $59 \mathrm{~mm}$ and $62 \mathrm{~mm}$, respectively. Constrained by the mechanical structure, the length of $\mathrm{BE}$ is less than $30 \mathrm{~mm}$ and the deformation $(\Delta \mathrm{L})$ of the fitted spring is less than $16 \mathrm{~mm}$. Analysis of the torque performance was conducted through calculation on the basis of the model presented in section 2. According to the different positions of the pivot, the torque characteristic curves of the VSA are divided into three parts and be presented in Figure 4. When the distance $(\mathrm{H})$ between pivot (D) and the center of rotation of the actuator $(\mathrm{O})$ is 0 to $30 \mathrm{~mm}$, the maximum allowable deflection angle between the driving disc and the output link is $\pm 0.5674 \mathrm{rad}$, and the maximum output torque in compliance mode is $0.5943 \mathrm{Nm}$. The output torque in the whole range of the allowable deflection angle is unsuitable to be applied in normal operation. When the distance is $55 \mathrm{~mm}$ to $62 \mathrm{~mm}$, the output torque is too large and the achievable maximum deflection angle $\theta_{\mathrm{S}}$ is very small, i.e., the maximum output torque in compliance can reach up to $80.389 \mathrm{Nm}$, but the maximum allowable deflection angle is only $0.07414 \mathrm{rad}$. The suitable operation range between the pivot and the rotation center of the actuator is defined from $30 \mathrm{~mm}$ to $55 \mathrm{~mm}$. 


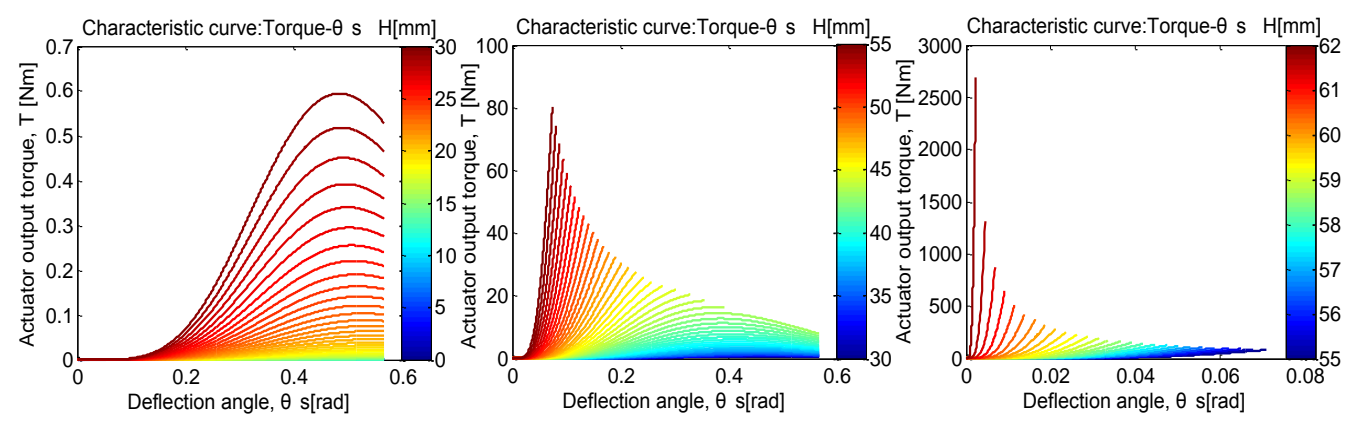
Figure 4. Torque Characteristic of the First VSA for the Quadratic
Spring Configuration

In Figure 4, when the value of $\mathrm{H}$ is between $30 \mathrm{~mm}$ to $43 \mathrm{~mm}$, with the increase of the deflection angle $\theta_{\mathrm{S}}$, the output torque of the actuator first increases and then decreases. This feature is determined by the mechanical structure of the actuator, and will be beneficial for the unexpected collision safety with the environment or human beings. The stiffness characteristic curves of the VSA are shown in Figure 5. When the pivot is located relatively far away from the center of rotation, the output stiffness is very large, even up to infinity, and this situation will help to improve the positioning accuracy.
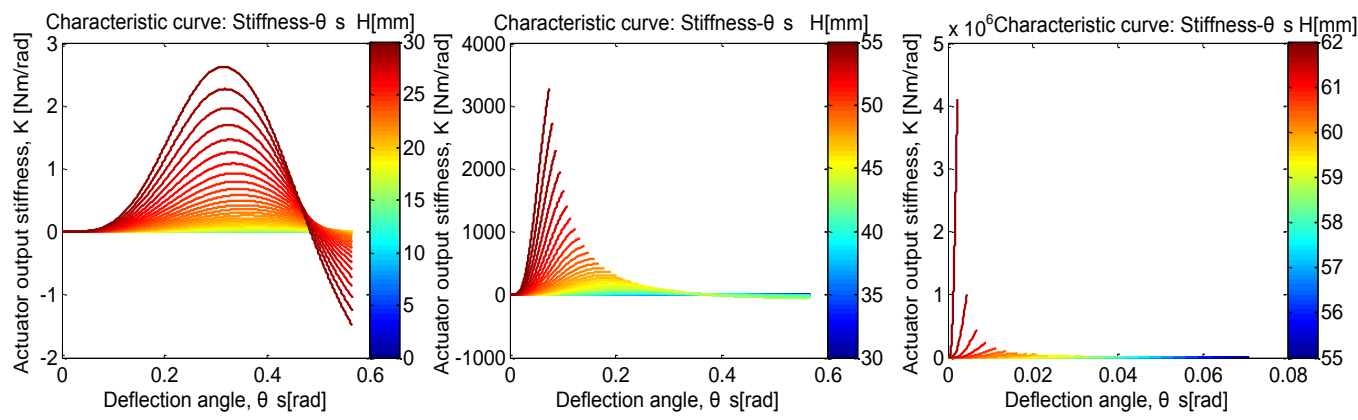

\section{Figure 5. Stiffness Characteristic of the First VSA for the Quadratic Spring Configuration}

In order to research the real stiffness characteristics under the piecewise linear spring group, the position of the pivot is set to be $30 \mathrm{~mm}, 35 \mathrm{~mm}, 40 \mathrm{~mm}, 45 \mathrm{~mm}$, $50 \mathrm{~mm}$, and $55 \mathrm{~mm}$, respectively. As is shown in Figure 6, the output stiffness curve corresponding to the actual piecewise linear spring group is interrupted curved segment. These curved segments follow the smooth stiffness curve. The discontinuous black curve segments represent the actual output stiffness curve. The blue curve represents the stiffness using the fitted quadratic spring. The red curve segments represent the difference between the actual stiffness and the fitted stiffness. The stiffness differences are relatively small. 

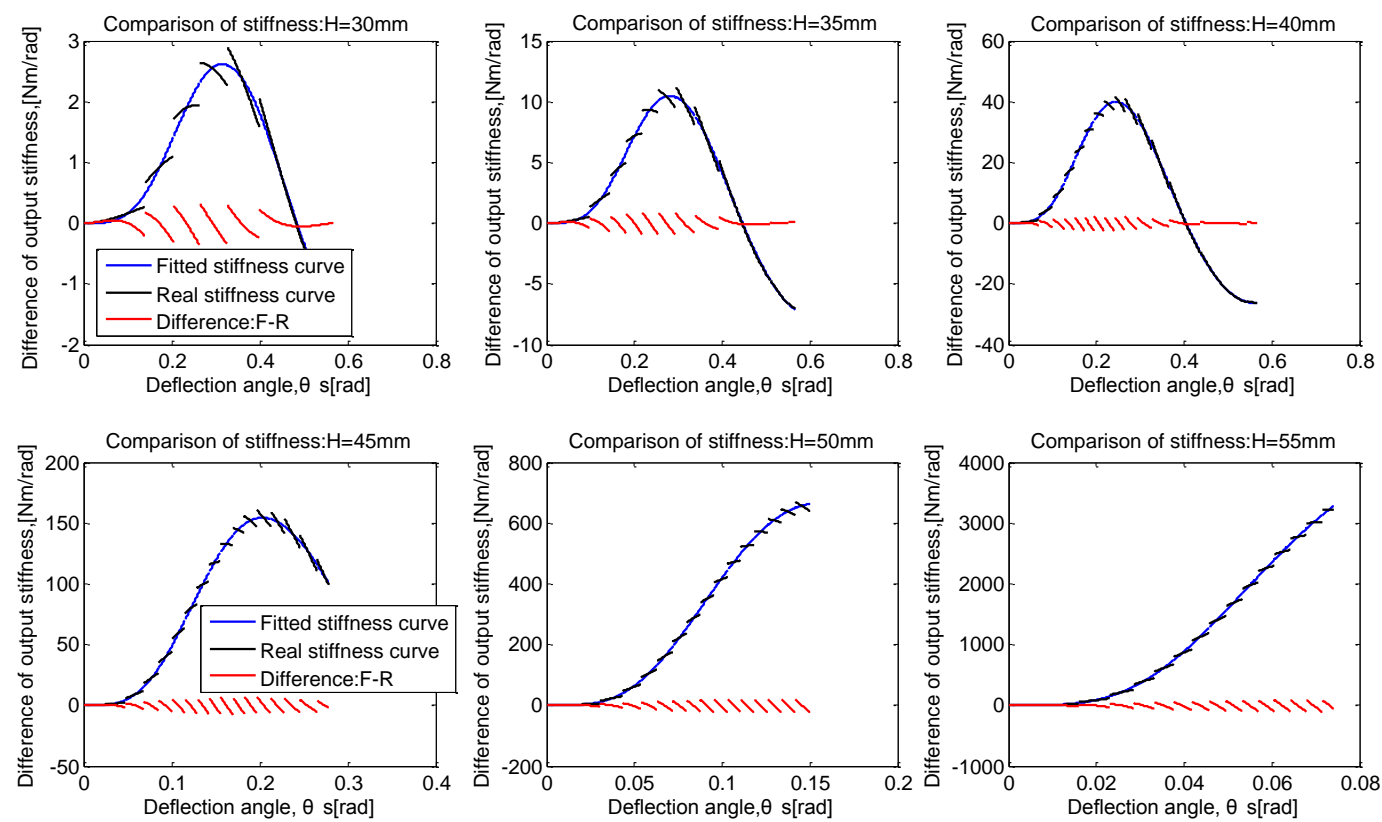

\section{Figure 6. Stiffness Characteristics of the First VSA for Piecewise Linear Spring Group Configuration}

\subsection{Comparison of the Spring Configuration}

To evaluate the promising advantage in the performance improvement of the proposed fitting quadratic spring, simulation studies were carried out. Figure 7 shows the differences of torque and stiffness for different types of spring configuration (linear and quadratic). Three positions of the pivot are chosen for analysis, i.e., $\mathrm{H}=30 \mathrm{~mm}$, $\mathrm{H}=35 \mathrm{~mm}$, and $\mathrm{H}=40 \mathrm{~mm}$. The other positions of the pivot also have the same stiffness difference. The black curve corresponds to the output stiffness or torque for using the quadratic spring. The blue circles represent the extreme value of output torque or stiffness. The torque or stiffness curves first increase and then decrease, which is determined by the mechanical structure of the VSA. When using the quadratic spring, the deflection angle $\theta_{\mathrm{S}}$ corresponding to the extreme stiffness or torque value has moved backwards. This feature indicates that the capacity for withstanding the external torque load is improved. For the quadratic spring configuration, when the pivot is located in $\mathrm{H}=35 \mathrm{~mm}$, the maximum elastic torque is $2.22 \mathrm{Nm}$, and the deflection angle $\theta_{\mathrm{S}}$ corresponding to extreme torque value is $0.4458 \mathrm{rad}$. If the linear springs with different stiffness were used, the deflection angle $\theta_{\mathrm{S}}$ corresponding to extreme torque value is $0.407 \mathrm{rad}$, which is smaller than 0.4458rad. Similarly, when the pivot is located in $\mathrm{H}=40 \mathrm{~mm}$, compared to the use of linear spring, the quadratic spring can increase the deflection angle range corresponding to the extreme output torque value. 

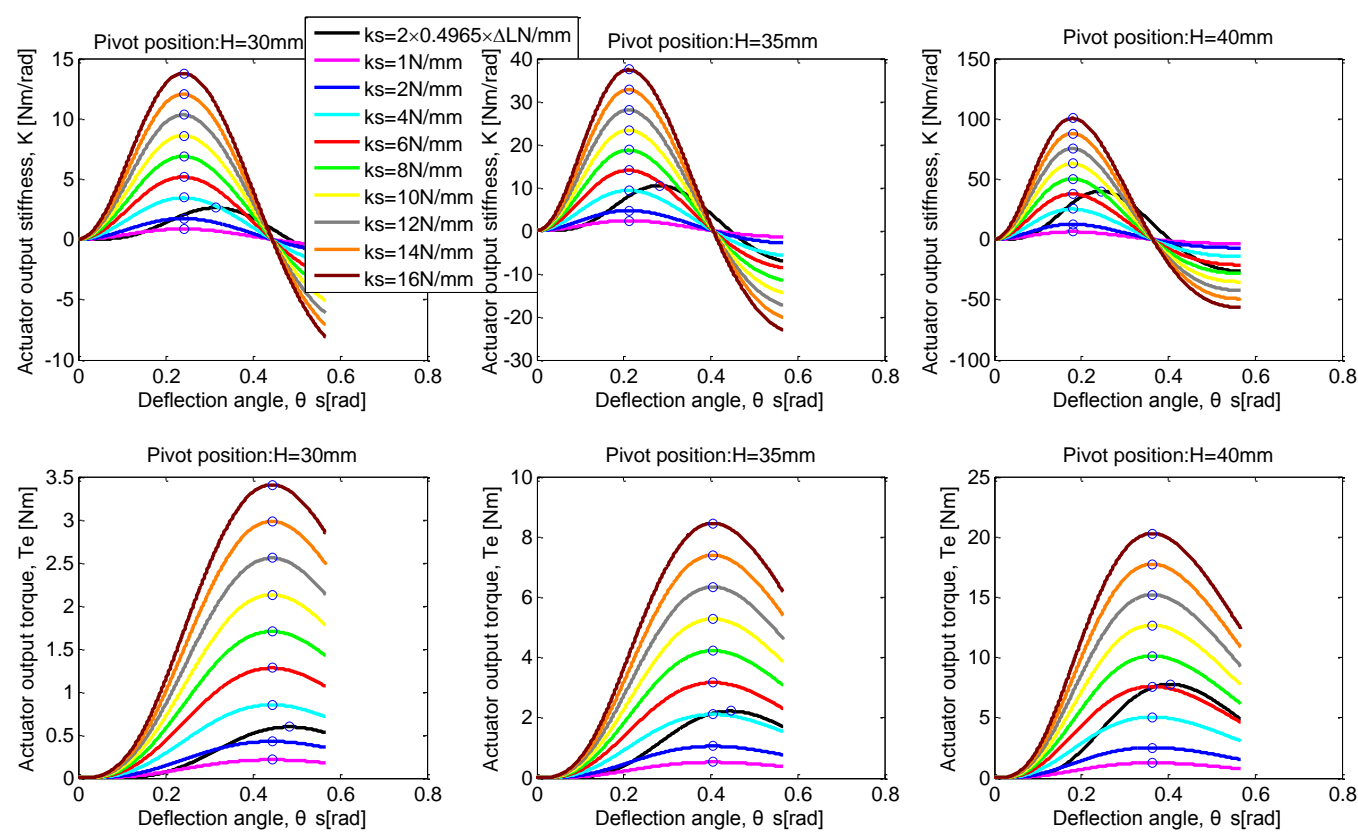

Figure 7. Comparison of the Stiffness and Torque Characteristics
between the Linear Spring Configuration and the Quadratic Spring
Configuration

Many different parameters can be used to describe the performance of the VSA system. The deflection angle range is an important measure of the compliance. By using the quadratic spring, the compliance range of the actuator is increased when the exerted torque is within the range of normal operation. Therefore, the working characteristic of the actuator is improved. When the actuator be subjected to an unexpected collision force or the applied external torque exceeds the threshold value, the torque or stiffness of the actuator will drop, which is beneficial for coping with the unpredictable interactions within not well defined environments and work in close vicinity with the human.

To reduce the mechanism size and the complexity of the elastic element, the linear spring can also be used. Under the same conditions as pivot position and spring deformation, the output torque will be directly affected by the stiffness of the linear spring. The selection of the linear spring stiffness Ks mainly depends on the requirements of output torque and the security restriction of impact power in unexpected situation. To simplify the model and the situation, a single degree of freedom spring-mass system is used to describe this transient impact. Suppose that the concentrated mass is $\mathrm{M}$ with a certain damping c connected in parallel to the base of the actuator, $x_{0}$ and $v_{0}$ are the initial displacement and speed of the mass $\mathrm{M}$, respectively. The motion equation of the $\mathrm{M}$ can be expressed as $m \ddot{x}+c \dot{x}+k x=0$. The displacement $x$ can be described as follow.

$$
x(t)=\sqrt{x_{0}^{2}+\left(\frac{v_{0}+\xi \omega_{n} x_{0}}{\omega_{d}}\right)^{2}} e^{-\xi \omega_{n} t} \sin \left(\omega_{d} t+\arctan \left(\frac{\omega_{d} x_{0}}{v_{0}+\xi \omega_{n} x_{0}}\right)\right)
$$

The $\xi$ is the damping ratio, $\omega_{\mathrm{n}}$ is the natural frequency, $\omega_{\mathrm{d}}$ is the damping natural frequency. Given the spring rate Ks and the damping coefficient $\mathrm{c}$, the reaction force $\mathrm{F}$ and the transient impact power $P$ can be described by

$$
\left.\begin{array}{l}
F=c \dot{x}(t)+K_{S} x(t) \\
P=F \dot{x}(t)=F v(t)
\end{array}\right\}
$$


The relationship between $\mathrm{P}$ and $\mathrm{Ks}$ is shown in Figure 8. Suppose that the initial displacement $x_{0}=0$, the $v_{0}=2 \mathrm{~m} / \mathrm{s}$, the $\mathrm{M}=2 \mathrm{~kg}$, and the $\xi=0.3$. As is illustrated in Figure 8, the higher the spring stiffness Ks, the greater the load capacity of the VSA. Meanwhile, the transient impact power will increase which is not beneficial for collision safety. We choose the spring stiffness $K s=7 \mathrm{~N} / \mathrm{mm}$ by the trade-off.
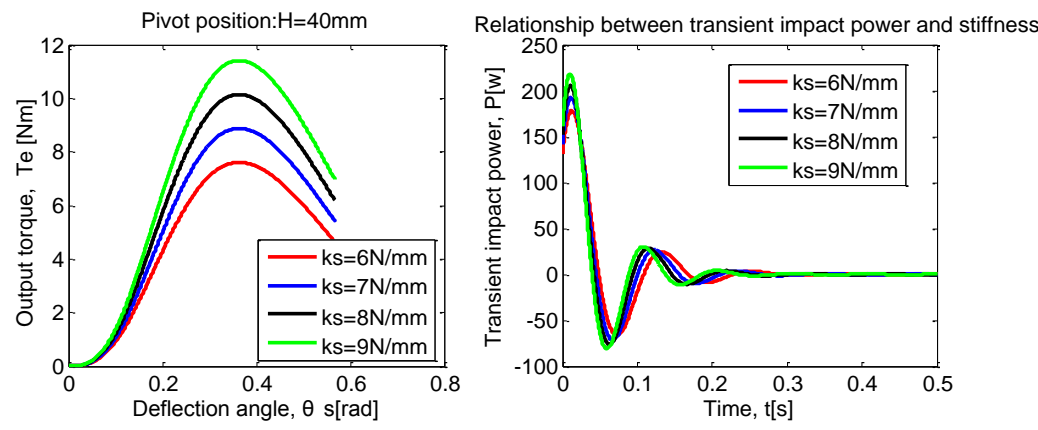

Figure 8. The Selection of the Linear Spring Stiffness Ks for the First VSA

\section{Mechanical Structure Solution of the First VSA}

The mechanical structure solution of the first VSA is shown in Figure 9. The conceptual prototype is essentially composed of a driving disc (1) functioning as the frame, where the variable stiffness mechanism components are located. The (1) is fixedly connected with the rotatable housing (25) through the screws. The (25) is connected with the fixed housing (23) by the bearing (22). The stiffness motor (21) is fixedly connected with the (25) through the bracket and screws. The output disc is connected with the (25) by the 4-point contact bearing (26). The screw transmission mechanism has self-locking function and consists of the bevel gear (18), threaded shaft (19), pivot movable base (10), and pivot movable base rail (11). The position motor (20) drives the spur gear (16) through the reducer and torque sensor (24). The internal gear (17) is fixed with the (25) by screws, and the motion of the (25) is realized by engaging the (17) and the (16). The (25) and the output link of the actuator are coaxial connected by the bearing (26). The spur gear (27) mounted on the output link meshes with the internal gear (29). The (29) is fixed with the (25). When the relative movement between the output link and the (25) occurred, the deflection angle $\theta_{\mathrm{S}}$ can be obtained by the encoder (28).

In addition, the (2) is diverting pulley and the (3) is the fixed baffles for the spring pedestal (4). The spring guide sleeve (5) is used to ensure the stability of the spring during working. When the spring group is compressed, the (4) will be moved along the guide groove (6). The (7) and (9) are the tendons on the left side and the right side, respectively. The (8) is the rotatable connection point between the lever (15) and the output disc. The (13) is the guide groove for pivot (12) in the lever (15), and the pivot (12) can be moved along the (13). The roller (14) is used to contact with the tendons and compresses the spring group. 


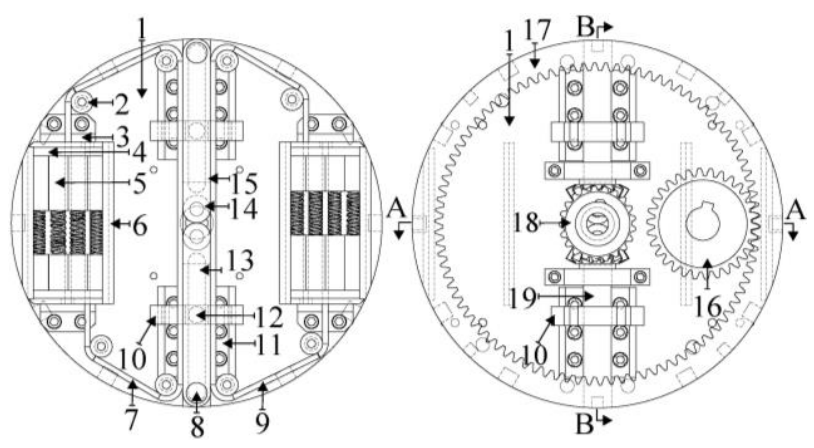

(a)
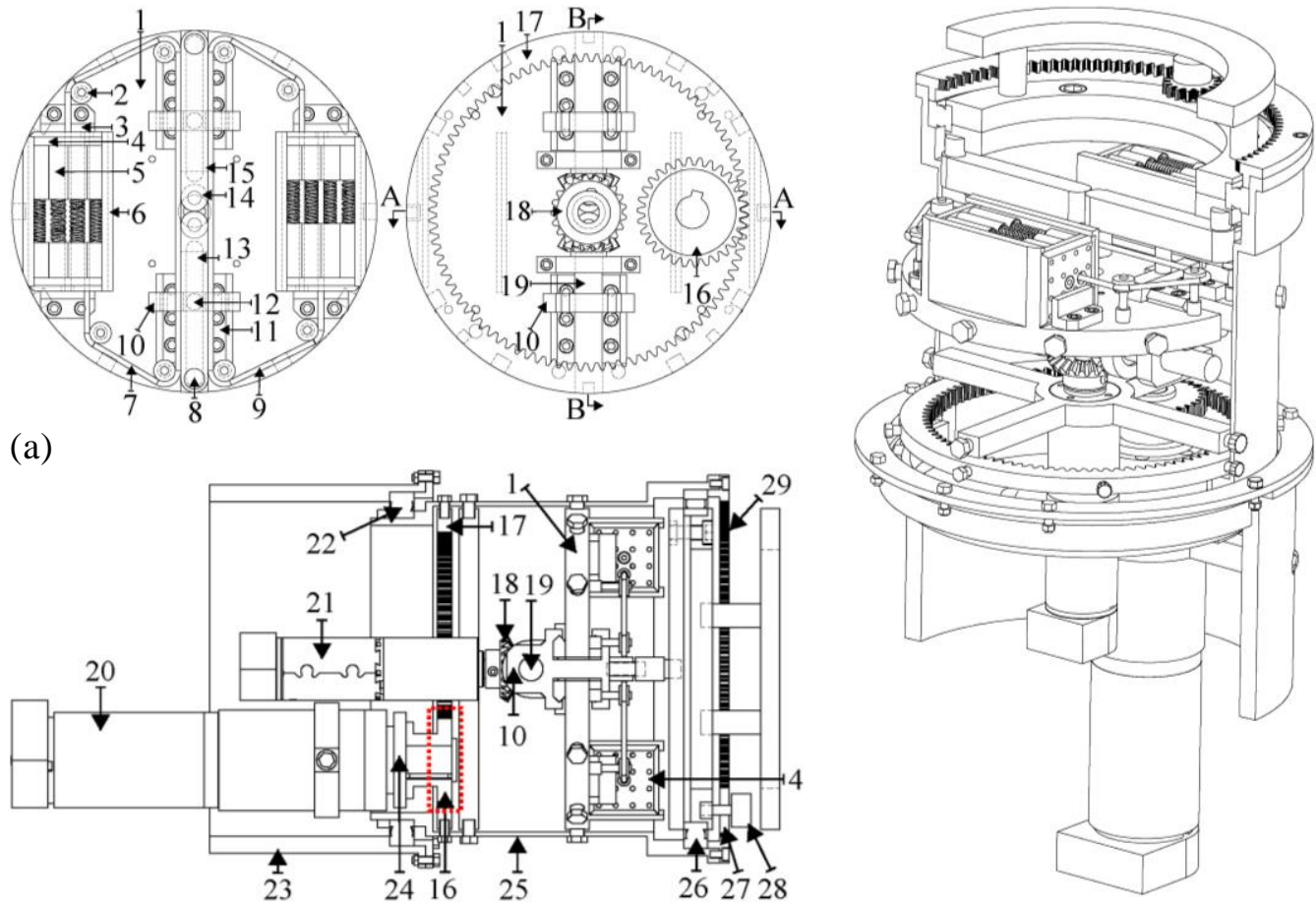

(b)

(c)

Figure 9. CAD Assembly Of The Actuator: A) The Front And Back View Of The Stiffness Adjustment Mechanism. B) A-A View Of The Actuator. C) Sectioned CAD View.

Figure 9 (c) demonstrates the feasibility of the conceptual VSA in mechanical design. The gap between the outer race of the spring and the guide sleeve is small, and there is a frictional damping force to suppress the oscillations of the springs. The allowable rotation range of the stiffness motor with respect to the fixed housing is limited by the cable attached to it. The allowable rotation angle range of the VSA is defined as $\pm 150^{\circ}$.

\section{Working Principle of the Second VSA}

\subsection{Conceptual Layout}

As mentioned above, in order to realize a variable transmission ratio between the internal elastic elements and the actuator output, a lever arm mechanism with a variable pivot point position is discussed in detail. It is noted that there are some other motheds based on lever arm mechanism can be used to change the transmission ratio. In this section, a VSA based on an adjustable moment arm mechanism is presented. The VSA is characterized by the property that the proportion of the two moment arms of the lever mechanism can be changed, and the variable stiffness feature is implemented by the connecting rod and slider mechanism. This is an energy efficiency way to regulate the stiffness because the output stiffness can be varied without changing the potential energy that is stored in the internal elastic elements. Moreover, the output stiffness is only related to the effective working radius for the linear spring configuration, but not to the exerted torques or the deflection angle. This feature makes the stiffness control more convenient. The working principle of the VSA is shown in Figure 10. 


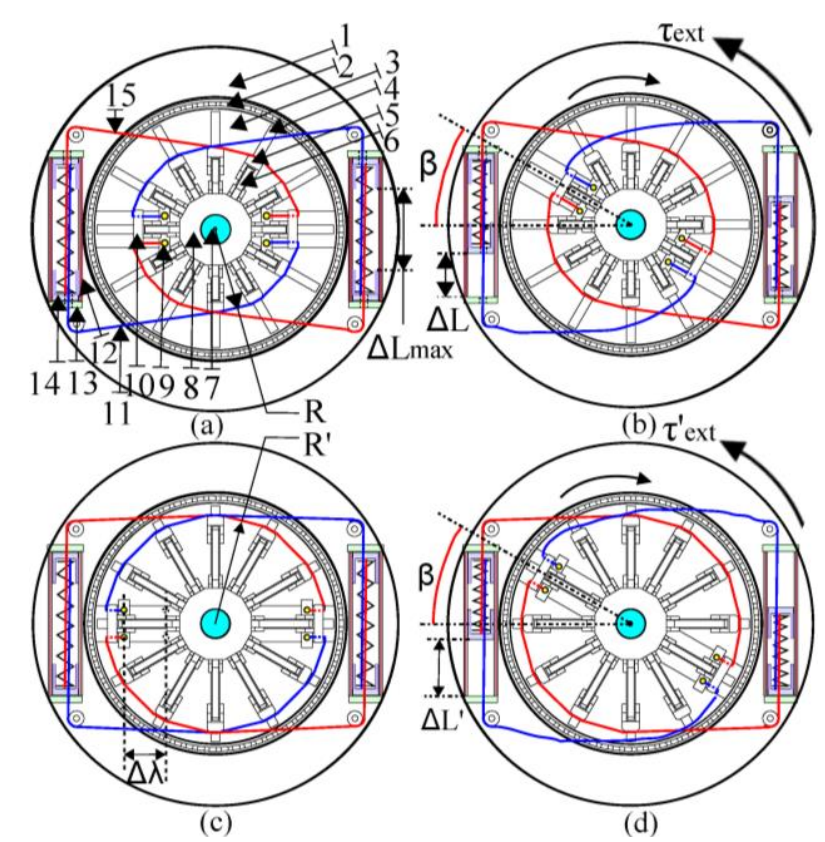

Figure 10. Conceptual Diagram Of The Second VSA: (A) Initial State. (B) Rotate An Angle Under The Action Of External Torque. (C) Radius Control Of The Driving Disc. (D) Rotate A Same Angle After Changing The Radius.

Figure 10 (a) demonstrates the initial state of this VSA. The compression spring is located inside the spring pedestal (14). The two red tendons are in the same plane, while the other two blue tendons are in another plane. The vertical distance between the two planes is small. One end of the connecting rod (6) driven by the threaded shaft (7) is used for connecting the nut (8) by the rotatable pin and the other end is connected with the slider (5). The (5) can be moved along the guide rod (4). The outer edge contours of the sliders form an approximate circular shape. The approximate circular contour is more nearly ideal circular if more sliders are used. The moveable point (9) and movable slider (10) are used to compensate the length variation of the tendons caused by the movement of the sliders. As is shown in Figure 10(b), Figure 10(c), and Figure 10(d), when the working radius of the driving disc (3) is changed from $\mathrm{R}$ to $\mathrm{R}^{\prime}$, in the same deflection angle $\beta$, the deformation of the spring is changed from $\Delta \mathrm{L}$ to $\Delta \mathrm{L}^{\prime}$, i.e., $\mathrm{R}<\mathrm{R}^{\prime}$ and $\Delta \mathrm{L}<\Delta \mathrm{L}^{\prime}$. The (9) connected with the tendon is moved along the radial direction of the (3) at the same time, and the moving distance is $\Delta \lambda$. The relationship between the $\Delta \lambda$ and the radius increment $\Delta R\left(\left|R-R^{\prime}\right|\right)$ is nonlinear. When the (3) is rotated clockwise, the red tendons are connected with the sliders and then the springs are compressed. The (14) is moved along the spring pedestal guide groove (12) and the (13) represents the fixed baffle for spring pedestal. Therefore, the output disc (1) is rotated and the blue tendons become slack at the same time. When the two spring guide sleeves contact with each other, the springs could not be compressed, and the actuator achieves the maximum degree of compliance respect to the current working radius. The output stiffness can be adjusted by changing the position of the sliders on the (4) and do not need to apply preload force on the spring. In Figure 10 (a), the tendon (11) and tendon (15) are respectively used for counter-clockwise rotation and clockwise rotation of the VSA. The (1) and the (3) is coaxially connected by the 4-point contact bearing (2). 


\subsection{Mathematical Modeling Of The Second VSA}

As shown in Figure 10 (b), suppose that the VSA is at its equilibrium position and the torque $\tau_{\text {ext }}$ is applied at the output disc. The deflection angle between the driving disc (3) and output disc (1) is $\beta$. The working radius is $\mathrm{R}$ and the spring rate is Ks. The springs are displaced by $x_{\mathrm{s}}$, and the torque and stiffness of the VSA are given by

$$
\left.\begin{array}{l}
T=2 F_{S} R=2 K_{S} x_{S} R=2 R^{2} \beta K_{S} \\
K=\frac{\partial T}{\partial \beta}=2 K_{S} R^{2}
\end{array}\right\} .
$$

It is noted that the stiffness $\mathrm{K}$ is a function of working radius $\mathrm{R}$ and spring stiffness Ks. If the linear spring is applied in the actuator, the output stiffness $\mathrm{K}$ depends on the working radius $\mathrm{R}$. If the purely quadratic spring (i.e., $\mathrm{F}(x)=a x^{2}$ ) configuration is used, the output torque and stiffness at the elastic transmission mode are formulated as

$$
\left.\begin{array}{l}
T^{\prime}=2 a R^{3} \beta^{2} \\
K^{\prime}=\frac{\partial T^{\prime}}{\partial \beta}=4 a R^{3} \beta
\end{array}\right\} .
$$

In addition, the calculation formulas of the torque and stiffness for cubic spring configuration (i.e., $\mathrm{F}(x)=b x^{3}$ ) are shown as follows.

$$
\left.\begin{array}{l}
T^{\prime \prime}=2 b R^{4} \beta^{3} \\
K^{\prime \prime}=\frac{\partial T^{\prime \prime}}{\partial \beta}=6 b R^{4} \beta^{2}
\end{array}\right\}
$$

As is illustrated in Figure 10 (c), the role of the movable point (9) is to ensure that the tendons always maintain contact with the sliders when the effective working radius is changed. The relationship between the moving distance $\Delta \lambda$ of the movable point (9) and the radius increment $\Delta \mathrm{R}$ is shown in Figure 11.

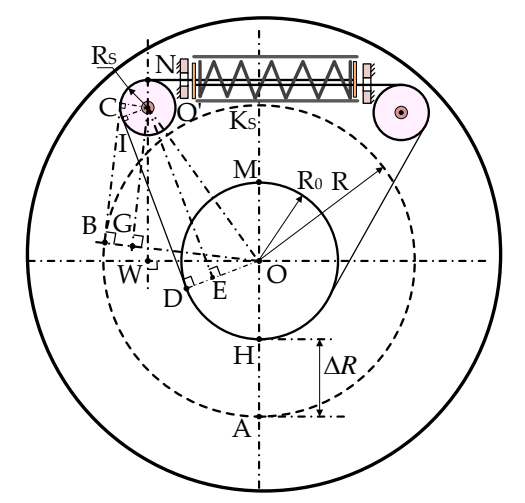

\section{Figure 11. Relationship Between The $\Delta \lambda$ And The Radius Increment} $\Delta r$

An ideal circular is used to replace the actual approximate circular contour in the calculation. Suppose that the two ends of the spring are connected with the tendon, the length of the spring could not be changed during changing the radius. The spring stiffness is Ks and the radius of the diverting pulley is Rs. The center of rotation of the diverting pulley is $\mathrm{O}^{\prime}$ point. The center of rotation of the VSA is $\mathrm{O}$ point. When the working radius is changed from $\mathrm{R}_{0}$ to $\mathrm{R}$, the tangent point 
between the tendon and diverting pulley is changed from point I to point C. Suppose that the $|\mathrm{OW}|$ and $\left|\mathrm{WO}^{\prime}\right|$ are known in mechanical structure solution. The relationship between the tendon length compensation $\Delta \lambda$ and the radius increment $\Delta \mathrm{R}$ is as follows.

$$
\left.\begin{array}{l}
\angle D O H=\frac{\pi}{2}-\angle O^{\prime} O E+\angle O^{\prime} O W=\frac{\pi}{2}-\arccos \frac{R_{0}-R_{S}}{{\sqrt{\overline{O W}^{2}+\overline{W O}^{\prime}}}^{\prime}}+\arccos \frac{\overline{O W}}{\sqrt{\overline{O W}^{2}+\overline{W O}^{2}}} \\
N I=R_{S} \angle N O^{\prime} I=R_{S} \angle M O D=R_{S}(\tau-\angle D O H) \\
\overline{I D}=\sqrt{\overline{O W}^{2}+\overline{W O}^{2}+\left(R_{0}-R_{S}\right)^{2}} \\
D H=R_{0} \angle D O H
\end{array}\right\}
$$

Therefore, the length of the tendon from point $\mathrm{K}$ to point $\mathrm{H}$ is $L_{R_{0}}=N I+\overline{I D}+D H$. When the working radius is changed from $R_{0}$ to $R$, the amount of displacement $\Delta \lambda$ is described as

$$
\begin{aligned}
& \overline{O^{\prime} G}=\sqrt{\overline{O W}^{2}+{\overline{W O^{\prime}}}^{2}-\left(R-R_{S}\right)^{2}} \\
& \angle D O B=\angle O^{\prime} O E-\arccos \frac{R-R_{S}}{\sqrt{\overline{O W}^{2}+\overline{W O}^{2}}} \\
& \angle M O B=\pi-\angle D O B-\angle D O H \\
& A B=R(\angle D O B+\angle D O H) \\
& N C=R_{S}(\pi-\angle D O B-\angle D O H) \\
& \overline{B C}=\overline{O^{\prime} G} \\
& L_{R}=A B+\overline{B C}+N C \\
& \Delta \lambda=L_{R}-L_{R_{0}}=f(R)
\end{aligned}
$$

In the tendon length compensation mechanism, a screw transmission mechanism with self-locking function is designed. The related design is demonstrated in Figure 12.

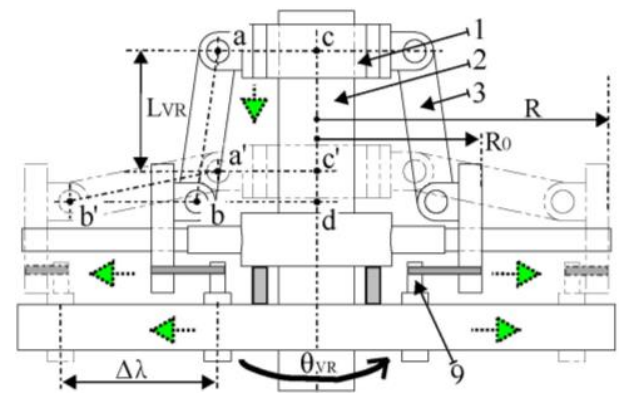

(a)

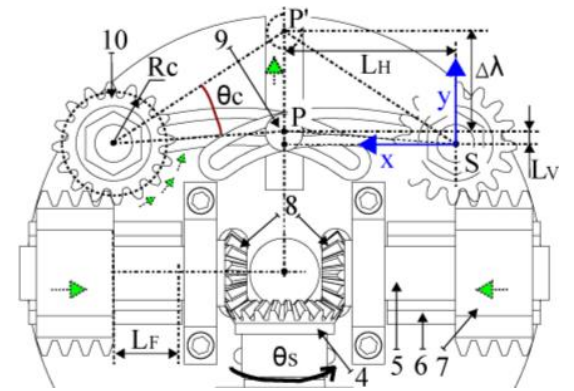

(b)

Figure 12. A) Working Radius Changing Mechanism for the Second VSA. B) Tendon Length Compensation Mechanism for the Second VSA.

Suppose that the initial radius is $\mathrm{R}_{0}$. The output angle of the stiffness motor is $\theta$ and the ratio of the reducer is $\eta$. The large bevel gear (4) and the threaded shaft (2) used for changing the working radius are driven by the same motor. The displacement of the rack (7) along the rail (6) is $\mathrm{L}_{\mathrm{F}}$. The rotation angle of the spur gear (10) used for driving the special contour frame is $\theta_{\mathrm{C}}$. The amount of 
displacement of the nut (1) is $L_{V R}$. The length of the connecting rod (3) is $|a b|=\left|a^{\prime} b^{\prime}\right|$. The pitch of the nut (1) is $P_{V R}$. In (2), the type of the screw thread is right-hand and trapezoidal. The pitch of the threaded shaft (5) is $\mathrm{P}_{\mathrm{F}}$ and the type of the screw thread is left-hand. $R_{C}$ is the pitch radius of the (10). The center of the moveable point (9) is $\mathrm{P}$ point and the center of the rotation of the (10) is $\mathrm{S}$ point. The $P$ point is also the initial position of the (9). The numbers of teeth of the (4) and Small bevel gear (8) are $z_{1}$ and $z_{2}$, respectively. The radial displacement of the (9) is $\Delta \lambda$ which corresponds to the rotation angle $\theta_{\mathrm{C}}$ of the (10). A Cartesian coordinate system is established. Finally, the equation describing the special contour for driving the (9) can be expressed as follows.

$$
\begin{aligned}
& L_{V R}=\frac{P_{V R}}{2 \pi} \theta_{V R}=\frac{P_{V R}}{2 \pi} \frac{\theta}{\eta}=\frac{P_{V R}}{2 \pi} \theta_{S}=\frac{P_{V R}}{2 \pi} \frac{2 \pi z_{2} \theta_{C} R_{C}}{z_{1} P_{F}}=\frac{P_{V R}}{2 \pi} f\left(\theta_{C}\right)
\end{aligned}
$$

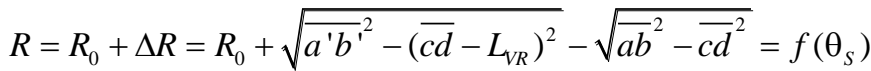

$$
\begin{aligned}
& \Delta \lambda=f(R)=f\left(\theta_{C}\right) \\
& x=\rho \cos \left(\theta_{C}+\arcsin \frac{L_{V}}{\sqrt{L_{V}{ }^{2}+L_{H}{ }^{2}}}\right) \\
& y=\rho \sin \left(\theta_{C}+\arcsin \frac{L_{V}}{\sqrt{L_{V}{ }^{2}+L_{H}^{2}}}\right) \\
& \rho=\sqrt{\Delta \lambda^{2}+L_{V}{ }^{2}+L_{H}{ }^{2}-2 \Delta \lambda \sqrt{L_{V}{ }^{2}+L_{H}{ }^{2}} \cos \left(\pi-\arcsin \frac{L_{H}}{\sqrt{L_{V}{ }^{2}+L_{H}{ }^{2}}}\right)}
\end{aligned}
$$

\section{Characteristics of Torque and Stiffness of the Second VSA}

In this section, the characteristics of torque and stiffness of the actuator for different types of spring configurations (linear, quadratic, and cubic) are shown in Figure 13.
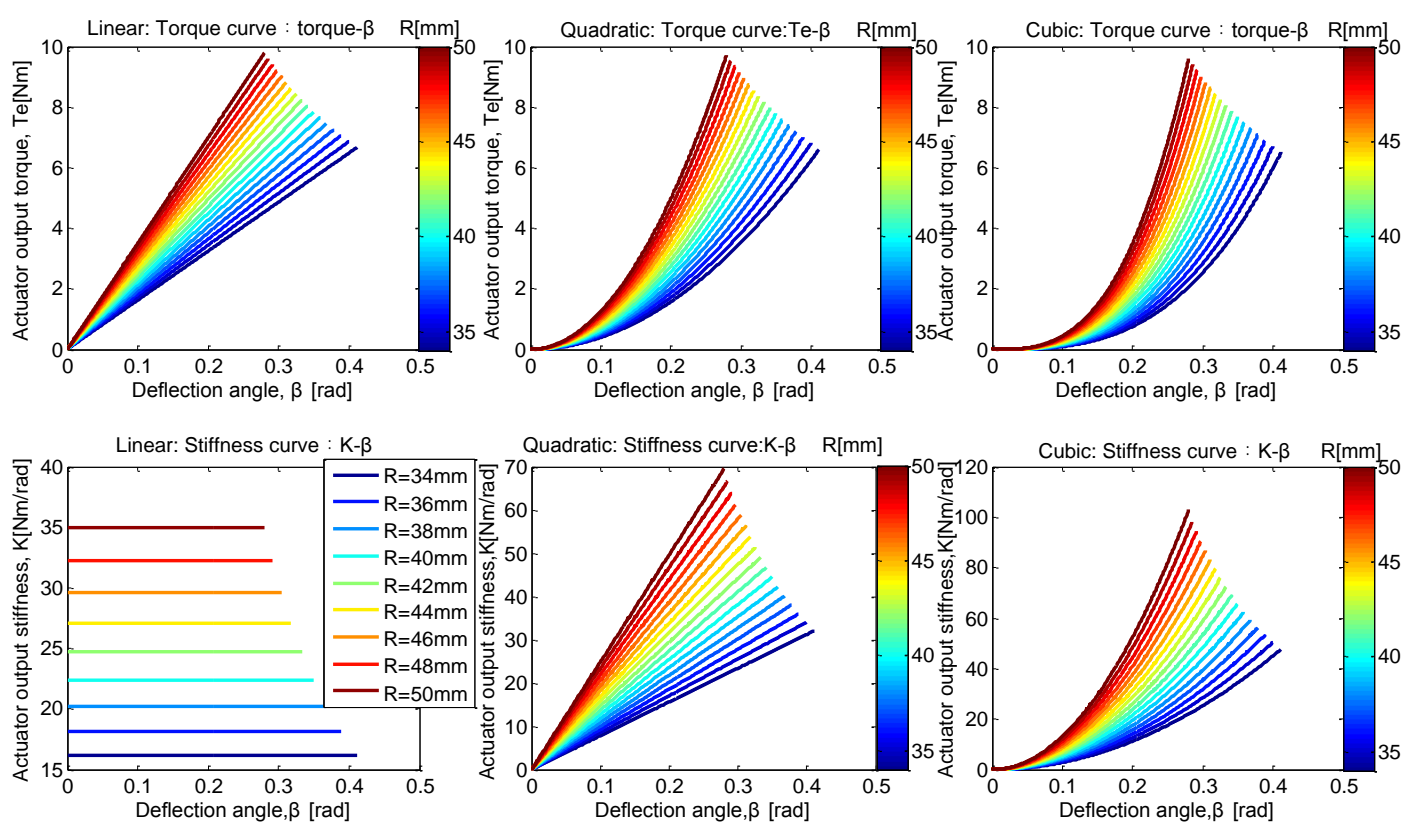

Figure 13. Stiffness Characteristics Of The Second VSA For Different Types Of Spring Configurations (Linear, Quadratic And Cubic) 
In the mechanical solution, the allowable maximum deformation of the spring is $14 \mathrm{~mm}$. The linear spring stiffness is $7 \mathrm{~N} / \mathrm{mm}$. The variation range of the working radius is from $34 \mathrm{~mm}$ to $50 \mathrm{~mm}$. The number of the sliders is 30 . The force-length relationship of the quadratic and cubic spring used in the calculation is $\mathrm{F}(x)=$ $0.4965 x^{2}$ and $\mathrm{F}(x)=0.035 x^{3}(x / m m, F / N)$, respectively. The mechanically allowable stiffness range at the elastic transmission of the VSA is from 16.184 $\mathrm{Nm} / \mathrm{rad}$ to $35 \mathrm{Nm} / \mathrm{rad}$ for linear spring configuration. The output stiffness $\mathrm{K}$ is kept constant in the allowable deflection angle range if the working radius is unchanged. It was shown that among linear, quadratic, and cubic springs, the linear spring configuration exhibits the easiest method for controlling the output stiffness. It is important to note that by using the linear spring, the desired output stiffness can be controlled in real-time and is not affected by the exerted torque or the deflection angle. The desired stiffness can be achieved not through the control of the spring pretension but by controlling the working radius. The energy consumption for stiffness regulation is mainly from the frictions, impacts and motion of the motor. This is an energy efficient actuator. Moreover, for the linear spring configuration, similarly to [6], this VSA is capable of minimizing energy consumption through exploiting the natural dynamics for fixed and variable frequency motions.

\section{Mechanical Structure Solution of the Second VSA}

The mechanical structure solution of the second VSA is presented in Figure 14. The processes of the stiffness regulation and position control are described as follows.
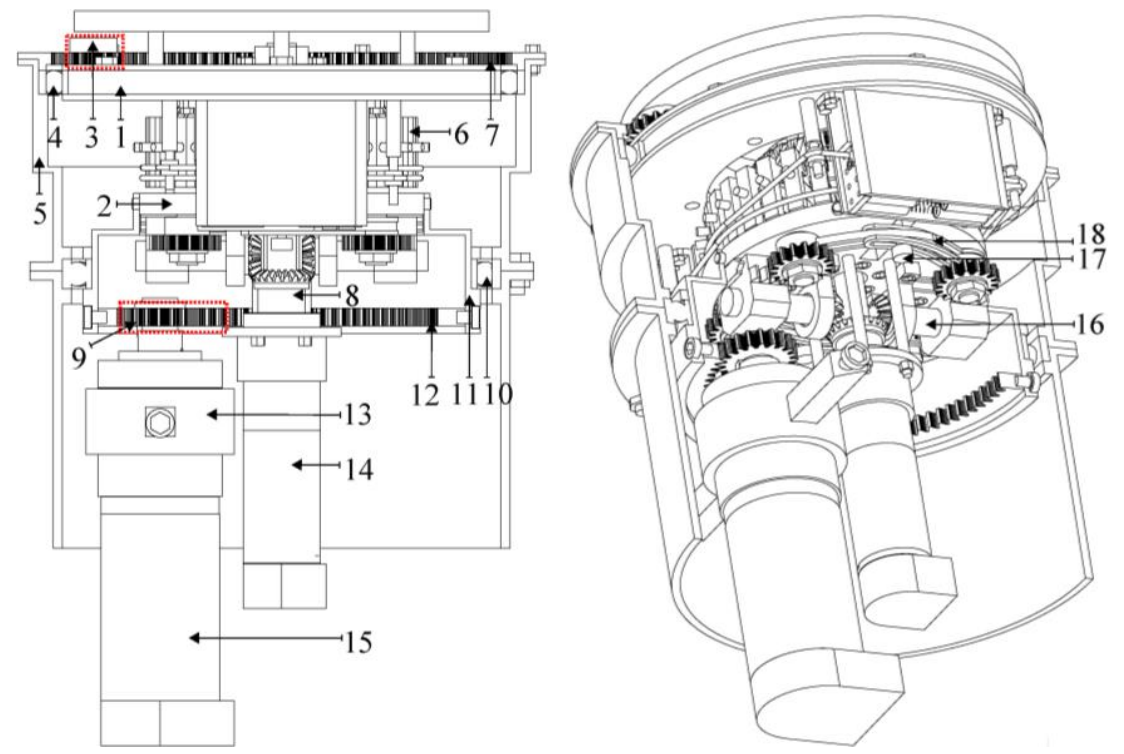

Figure 14. CAD Assembly of the Second VSA

The variable stiffness mechanism components are located in the driving disc (2). The stiffness motor (14) is fixedly connected with the (2) by the bracket. The (14) drives the bevel gear (8) through the reducer, and the rotational motion of the threaded shaft (16) is achieved by the engagement between the two bevel gears. The motion of the special contour frame (18) is realized by the meshing of spur gear and rack. The movable point (17) is driven by the two special contour frames. The nut for driving the connecting rod is driven by the (8). The connecting rod drives the sliders (6) and then the working radius is changed. The 
displacement range of the slider along the guide rod is restricted by the mechanical block. The rotatable housing (11) is fixed with the internal gear (12) by the screws. The (11) is connected with the fixed housing (5) by the bearing (10). The position motor (15) is fixed with the (5) by the bracket (13). The actuation of the (11) is realized by engaging the ring gear (12) and spur gear (9). The internal gear (7) is fixed with the (5), and the (1) is connected with the (5) by the 4-point contact bearing (4). The encoder (3) driven by the spur gear is used to calculate the deflection angle between the output disc (1) and the driving disc (2).

\section{Conclusions and Future Works}

In this paper, two types of novel variable stiffness actuator based on the adjustable moment arm mechanism were presented in which the stiffness can be regulated with minimum energy consumption. The two actuation systems can simultaneously and independently control the output position and output stiffness. In the first VSA, compared with the existing variable stiffness actuators that based on the variable lever arm principle, the way of the spring compressed is more convenient to be implemented by using the tendon and pulley mechanism. Furthermore, this spring compression method is more beneficial for dealing with the unexpected dynamic collision situation. The internal elastic elements of the VSA use the fitted quadratic spring, which improve the stiffness and torque characteristics of the actuator. The stiffness regulation of the second VSA is realized by implementing the connecting rod and slider mechanism. This is a equivalent model of the lever arm mechanism with a variable length moment arm. The stiffness is only related to the effective working radius for the linear spring configuration. This feature makes the stiffness control is much more convenient. So we do not need to design the complicated control strategy for achieving the desired output stiffness. The conceptual layout and working principle of the two actuators are elaborated and the characteristics of actuating torque and output stiffness for different spring configurations are demonstrated. The mechanical structure solutions of the two actuators are described.

Future works will include the research on the torque and stiffness control strategies and the finite element simulation analysis of the mechanical structure.

\section{Acknowledgements}

This work is supported by the National High Technology Research and Development Program of China (Grant Number: 2009AA04Z220 and 2006AA040206).

\section{References}

[1] Jung-Jun Park, Byeong-Sang Kim, Jae-Bok Song and Hong-Seok Kim. Safe link mechanism based on nonlinear stiffness for collision safety. Mechanism and Machine Theory, 43 (2008).

[2] Amir Jafari. Variable Impedance Actuators. Available from: http://www.birl.ethz.ch/sssr2012/onlinematerial-/Amir Jafari.pdf. (2012) August 14.

[3] R. Schiav i, G. Griol and S. Sen, A. Bicchi. VSA-II: a Novel Prototype of Variable Stiffness Actuator for Safe and Performing Robots Interacting with Humans. Proceedings of the 2008 IEEE International Conference on Robotics and Automation, (2008) May 19-23; Pasadena, CA, USA.

[4] Bram Vanderborght, Nikos G. Tsagarakis, Ronald Van Ham, Ivar Thorson and Darwin G. Caldwell. MACCEPA 2.0: compliant actuator used for energy efficient hopping robot Chobino1D. Autonomous Robots, 31, 1 (2011).

[5] B.-S. Kim and J.-B. Song. Hybrid Dual Actuator Unit: A Design of a Variable Stiffness Actuator based on an Adjustable Moment Arm Mechanism. Proceedings of the 2010 IEEE International Conference on Robotics and Automation, (2010) May 3-8; Anchorage, Alaska, USA.

a. Jafari, N. Tsagarakis, B. Vanderborght, and D. Caldwell. A Novel Intrinsically Energy Efficient Actuator With Adjustable Stiffness (AwAS). IEEE/ASME Transactions on Mechatronics, 18, 1 (2013).

[6] Jafari, N. Tsagarakis, B. Vanderborght, and D. Caldwell. AwAS-II: A New Actuator with Adjustable Stiffness based on the Novel Principle of Adaptable Pivot point and Variable Lever ratio. Proceedings of the 2011 IEEE International Conference on Robotics and Automation, (2011) May 9-13; Shanghai, 
China.

[7] N. G. Tsagarakis, I. Sardellitti, and D. G. Caldwell. A New Variable Stiffness Actuator (CompActVSA): Design and Modeling. Proceedings of the 2011 IEEE/RSJ International Conference on Intelligent Robots and Systems, (2011) September 25-30; San Francisco, CA, USA.

[8] Nikos C. Karavas, Nikos G. Tsagarakis and Darwin G. Caldwell. Design, Modeling and Control of a Series Elastic Actuator for an Assistive Knee Exoskeleton. Proceedings of the 4th IEEE RAS/EMBS International Conference on Biomedical Robotics and Biomechatronics, (2012) June 24-27; Roma, Italy.

[9] L. C. Visser, R. Carloni, R. Ünal, and S. Stramigioli. Modeling and Design of Energy Efficient Variable Stiffness Actuators. Proceedings of the 2010 IEEE International Conference on Robotics and Automation, (2010) May 3-8; Anchorage, Alaska, USA.

[10] S. Groothuis, G. Rusticelli, A. Zucchelli, S. Stramigioli, and R. Carloni. The vsaUT-II: a Novel Rotational Variable Stiffness Actuator. Proceedings of the 2012 IEEE International Conference on Robotics and Automation, (2012) May 14-18; Saint Paul, Minnesota, USA.

[11] M. Fumagalli, E. Barrett, S. Stramigioli, and R. Carloni. The mVSA-UT: A Miniaturized Differential Mechanism for a Continuous Rotational Variable Stiffness Actuator. Proceedings of the 4th IEEE RAS/EMBS International Conference on Biomedical Robotics and Biomechatronics, (2012) June 2427; Roma, Italy.

[12] R. Carloni, L. C. Visser, and S. Stramigioli. Variable Stiffness Actuators: A Port-Based Power-Flow Analysis. IEEE Transactions on Robotics, 28, 1 (2012).

[13] Ludo C. Visser, Raffaella Carloni, and Stefano Stramigioli. Energy-Efficient Variable Stiffness Actuators. IEEE Transactions on Robotics, 27, 5 (2011).

[14] S.A. Migliore, E.A. Brown, and S.P. DeWeerth. Biologically Inspired Joint Stiffness Control. Proceedings of the 2005 IEEE International Conference on Robotics and Automation, (2005) April; Barcelona, Spain.

[15] Datong Qin and Liyang Xie, Editor, Spring Design, Chemical industry press, Beijing (2013).

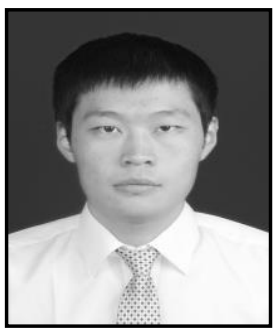

\section{Authors}

Jishu Guo He received the B.S. degree in mechanical designing and manufacturing automation from Shandong Jiaotong University, Jinan, China in 2009, and the M.S. degrees in mechatronic engineering from Shandong University, Jinan, China in 2012. He is now a Ph. $\mathrm{D}$ candidate in control theory and engineering of Shandong University. His research interests include design and control of variable stiffness actuators, robot arm and motion planning.

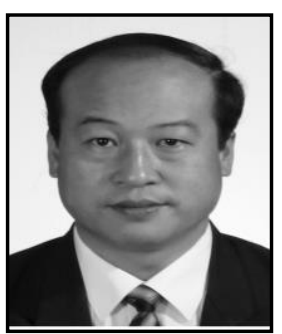

Guohui Tian He was born in August 1969 in Hebei, China. He is a professor in School of Control Science and Engineering, Shandong University. And also he is the member of Chinese Association for Artificial Intelligent Autonomous System. He received the B.S. degree from Department of Mathematics, Shandong University, Jinan, China, in 1990, the M.S. degree from the Department of Automation, Shandong University of Technology, Jinan, China, 1993, and the Ph.D. degree from School of Automation, Northeastern University, Shenyang, China, in 1997. He studied as a post-Doctorial researcher in School of Mechanical Engineering of Shandong University from 1999 to 2001, and studied as a visiting professor in Graduate School of Engineering of Tokyo University of Japan from 2003 to 2005. His research interests include service robot, intelligent space, collaboration and cooperation of multi-robot system. 
International Journal of Control and Automation Vol.8, No.8 (2015) 